\title{
Interfacial Stress Analysis of the Second-Reinforced Load-Bearing Beam Based on COD Criterion
}

\author{
Yushi Yin ${ }^{1,2, a}$ Yingfang Fan ${ }^{1, b}$ Yihong $X u^{1,2, c}$ \\ 1Dalian Maritime University Road and Bridge institute, Dalian 116000,China; \\ ${ }^{2}$ Liaoning Provincial College of Communications, Shenyang 110122, China. \\ ayys00080568@163.com, b35196002@qq.com, cxyh0756821@163.com
}

Keywords: Interfacial stress, COD, D-B model, shear stress.

\begin{abstract}
Since there exists plastic zone on the crack tip of reinforced beam, it is impossible to use crack instability criterion for brittle materials. Based on the theory of fracture mechanics, by using D-B model and considering the actual construction conditions, this study puts forward a COD criterion-based computing method to make a quantitative analysis of the shear stress of CFRP reinforced interface. The results show that it is feasible to use COD detection value to analyze the shear stress of FRP-to-concrete interface for those elasticity-dominant materials with small size of plastic zone $\left(\frac{\sigma_{s}}{\sigma_{0}} \leq 0.6\right)$. In actual construction, crack initiation detection $\operatorname{COD}\left(\delta_{c}\right)$ is simpler to use
\end{abstract} and easier to measure. It will prevent debonding and prompt the FRP-to-concrete interfacial debonding.

\section{Introduction}

According to the energy theory, the reason for crack propagation is that the synthetical value of stress and strain has reached a critical value[1,2,3]. It is feasible to discuss the unstable crack extension of brittle material from the point of stress changes. However, when the crack tip yields in a large scale, the standpoint of stress should be adopted to discuss the instability problem. The crack tip opening displacement (COD for short) is a variable of plastic strain at crack tip. It is believed that when the crack tip opening displacement (COD) reaches a critical value, crack expansion will occur, which is the essence of COD critierion[7-13].

\section{Research Background}

\subsection{The Establishment of the Mechanics Model to Reinforcement Loading Concrete Beam}

About bridge structure reinforcement which named loading-reinforcement on the ground of the objective conditions, such as traffic without interruption, time-condition, and many other reasons. In reinforcement engineering, the original beam body is basically with seam working, so the work which is a part of reinforcement is the "filled-gap", which filled the original bridge cracks, as a result of the limitation of objective and artificial filling method, and the traffic without interruption, so generate center cracks throughout the beam body, the volume of crack propagate $\Delta a$ in the center of the beam is much smaller than the beam height $h$, so the cracks in the body can be simplified as the model of the infinite center throughout plate, and based on the research purpose, take plain concrete beam as the research object, here does not affect the results of the study and according to the construction of the objective conditions, at the time of removing the old FRP, a new hydraulic jack to the beam generates vertical displacement, then we consider that: (1) Crack tip already from brittle fracture, into the plastic region, and the area is not big $\left(\frac{\sigma_{s}}{\sigma_{0}} \leq 0.6\right)$, the beam body deformation is small.(2) The beam body height is more than secondary cracking of crack, accord with D - B model of background model.(3) Section situation is in line with flat section assumption. As shown in figure 1 to 3 . 

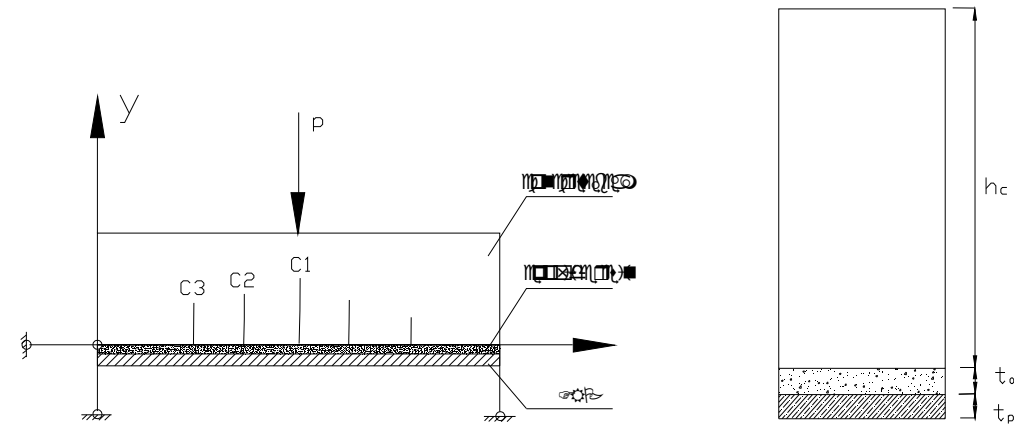

Fig. 1 The profile of reinforcement beam Fig. 2 The cross-section of reinforcement beam

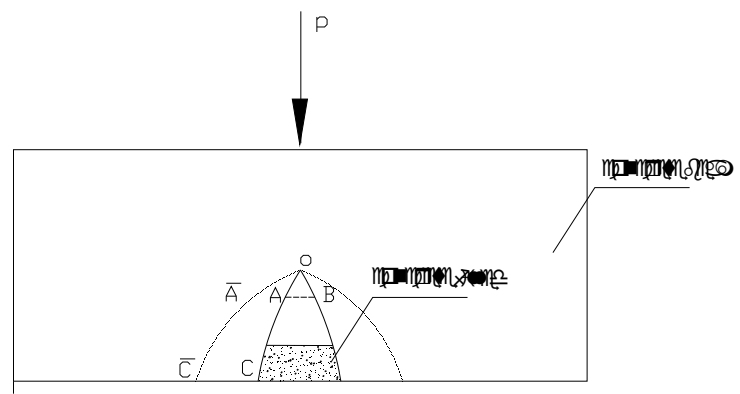

Fig. 3 The mechanics mode of crack beam through center infinity

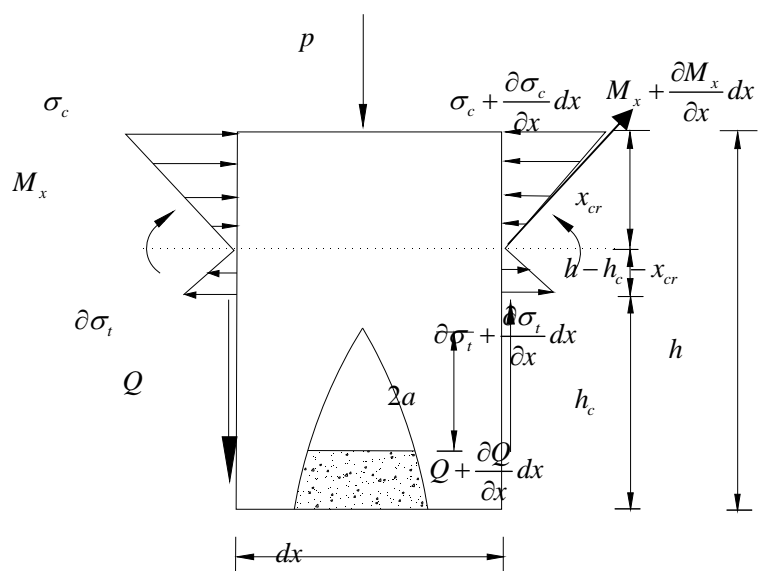

Fig. 4 Stress analysis diagram of main crack fracture of infinitesimal body

2.2 The Calculation of Model Parameters

\subsubsection{The Calculation of Pressure Zone Height}

According to the figure 5, strain geometric relationships:

$$
\begin{gathered}
\frac{\varepsilon_{c}}{\varepsilon_{t u}}=\frac{X_{c r}}{h-h_{c}-X_{c r}} \\
x_{c r}=\frac{\varepsilon_{c}\left(h-h_{c}\right)}{\varepsilon_{c}+\varepsilon_{t u}}
\end{gathered}
$$

\subsubsection{The Calculation of the Main Cracking Bending Cracks}

According to the main cracks $\sum m=0$ :

$$
\begin{aligned}
& \frac{1}{2} \sigma_{c} \cdot x_{c r} \cdot \frac{2}{3} x_{c r}-\frac{1}{2} f_{t} \cdot\left(h-x_{c r}\right) \cdot \frac{2}{3}\left(h-x_{c r}\right)-M_{c r}=0 \\
& M_{c r}=\frac{1}{3}\left[\sigma_{c} x_{c r}{ }^{2}-f_{t}\left(h-x_{c r}\right)^{2}\right] \\
& \sigma_{c}=E \varepsilon_{c}
\end{aligned}
$$


According to geometry relationship, in figure 1-4 infinitesimal body border $M=\frac{l-2 b}{l} M_{c r}$ (4)

According to the material mechanics basic knowledge:

$\sigma_{t}=\frac{\left(h-h_{c}-x_{c r}\right)}{I_{z}} M_{c r}$

$T_{c}=\frac{1}{2} \sigma_{c} \cdot x_{c r}$

$T_{t}=\frac{1}{2} \sigma_{t} \cdot\left(h-h_{c}-x_{c r}\right)$ (5)

After the reduction on the boundary, forming a main moment, the main vector:

$$
\left\{\begin{array}{l}
M=\frac{l-2 b}{l} M_{c r} \\
F_{R}=T_{c}-T_{t}
\end{array}\right.
$$

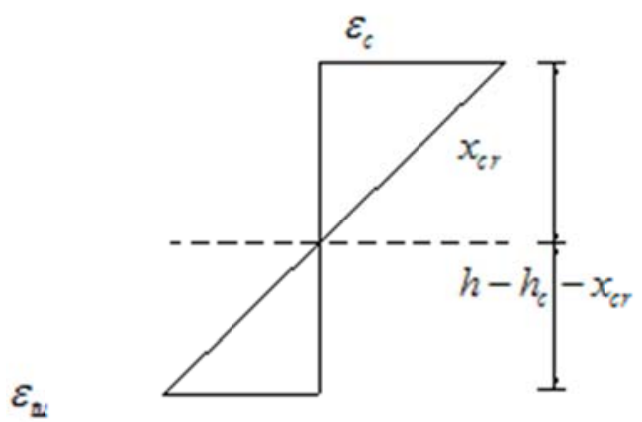

Fig.5 Strain diagram of main crack

\section{A Displacement Value Calculation of D-B Strip Yield Zone Model Based on the COD}

D-B model [7] can be used for processing elastic-plastic problems where there is center throughout the infinite thin plate under the uniform tensile stress. The model assumption that: the crack tip plastic region extends to other sides of the tip zone along the crack line, splitting shaped; Material in plastic zone take as the ideal elastic-plastic state, and the surrounding plastic zone is elastic; The boundary of plastic zone and elastic, existing the binding force $\sigma_{s}$, here $\sigma_{s}$ called the yield stress of concrete.

\subsection{Formula of Crack Opening Displacement COD $(\delta)$ under the Action of $F_{r}$}

According to the formula (5), there formed main vector $F_{R}$ in the interface, according to the cassette theorem of mechanics of materials, calculation expression of crack tip opening displacement $\delta$ of D-B model can be introduced [7] :

$\delta_{1}=\frac{8 \sigma_{s} a}{E \pi} \ln \sec \left(\frac{\pi \sigma^{\prime}}{2 \sigma_{s}}\right)$

$\sigma^{\prime}=\frac{F_{R}}{b h}$

The main vector $F_{R}$ influences $\delta_{1}$

3.2 Formula of Crack Tip Opening Displacement COD $(\delta)$ under the Action of $M$

The calculate method of COD under the action $M$ can be indirectly by literature [2]:.

$J=\int_{o}^{M}\left(\frac{\partial \theta}{\partial \boldsymbol{a}}\right) d m=\frac{2}{h-h_{c}} M^{2}$ 


$$
\begin{aligned}
& J=\sigma_{s} \cdot \delta_{2} \\
& \delta_{2}=J / \sigma_{s}
\end{aligned}
$$

\subsection{Formula of crack tip opening displacement COD $(\delta)$ under the action of $M_{\text {and }} F_{r}$}

According to the small deformation assumption and using the principle of superposition, under the action $M$ and $F_{r}$ :

$\delta=\delta_{1}+\delta_{2}$

According to the COD criterion when: $\delta=\delta_{c}$ crack generates, crack extends.

$\delta_{c}$ is the critical value of crack generates which can be determined by tests.

\section{Bottom displacement $\Delta l$ at main crack based on the theory of COD}

\subsection{The energy theory of Griffith}

Griffith's energy theory: when the crack extension of per unit area release strain energy is equal to the surface energy needed for the free surface, the crack is in opening state.namely $\Delta u=\Delta T$

$$
\begin{aligned}
& \frac{\pi \sigma_{0}^{2} \Delta S^{2}}{4 E b}=2 \Delta S \cdot r \\
& \Delta l=\frac{8 E r}{\pi \sigma_{0}^{2}}
\end{aligned}
$$

$\delta_{2}$ has been obtained, so ${\widetilde{\sigma^{\prime \prime}}}^{\prime \prime}=\frac{2 \sigma_{s}}{\pi} \arccos e^{-\left(\frac{\delta_{2} E \pi}{8 a \sigma_{s}}\right)}$

Unite Formula (6) $\sigma_{0}=\sigma^{\prime \prime}-\sigma^{\prime}$

Formula (11)with (10), can get bottom displacement of concrete beam variation $\Delta l \quad(\overline{\bar{C} C})$.

\section{The derivation of the main cracks on the edge of FPR}

For the main crack zone where generates strain $\varepsilon_{e}$ that is on edge of FRP and existing mature theoretical formula, using the method of literature [6] : $\tau_{a}=\frac{d \sigma_{f}}{d x} t_{f}$. For $t_{f}$ is sheet thickness; $d x$ is strain gage; $d \sigma_{f}$ is the tensile stress increment between FPR. Assuming bond destruction as the ideal state, so the slip of the epoxide resin is $s=\bar{c} c-\varepsilon_{e} \cdot l_{p}, l_{p}$ is longitudinal length of FRP. According to the Xinzheng Lu's [6] bond-slip constitutive equation:

$\left\{\begin{array}{l}\tau=\tau_{\max } \frac{s}{s_{0}} \\ \tau=\tau_{\max } \frac{s_{f}-s}{s_{f}-s_{0}} \\ \tau=0\end{array}\right.$ 


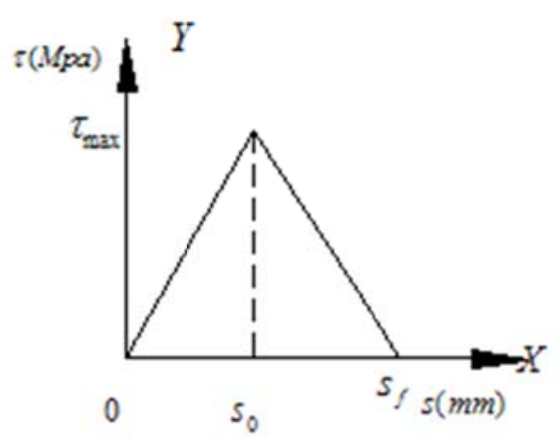

Fig.6 Relationship between slip and stress

$s_{0}=0.01453 f_{t} ; \quad s_{f}=1.7886 \frac{G_{f}}{f_{t}} ; \tau_{\max }=1.1182 f_{t}$ 。

$f_{t}$ is the tensile strength of concrete; $G_{f}$ is the shear modulus of the epoxide resin. Through the constitutive equations above different interfacial stresses can be calculated.

\section{Conclusion}

(1) Different from the conventional method of using micro unit mechanical equilibrium and differential equations to calculate bonding stress, this study puts forward for the first time the bonding constructive equation combined with COD in solution of interfacial stress in the concrete structure. This method is only feasible for those materials dominated by elastic zone and with small size of plastic zone $\left(\frac{\sigma_{s}}{\sigma_{0}} \leq 0.6\right)$. But this method does not work if the total cross section yields.

(2) In crack problems, crack initiation $\operatorname{COD}\left(\delta_{c}\right)$ is simpler to use and easier to measure. If this method is applied in acutal construction, it will prevent debonding and indicate the possiblility of the FRP-to-concrete interfacial debonding.

(3) This study mainly focuses on plain concrete beams and the formula is used for second-reinforced concrete beams without FRP and then stressed again with FRP. The result won't affect the nature of the study and its conclusions. The same study method could be taken when using the concrete concrete and prestressed concrete structure, and the formula could be adjusted.

\section{Acknowledgements}

The authors would like to thank the supports from the National Natural Science Foun-dation of C hina (Grant No. 51178069) .

\section{References}

[1] Hutchinson J W.Singular behaviour at the end of a tensile crack in a hardening material. Journal of the Mechanics and Physics of Solids .1968,p.231-237.

[2] Rice JR,Rosengren GF.Plane strain deformation near a crack tip in a power-law hardening material. Journal of the Mechanics and Physics of Solids.1968, p.105-112.

[3] Drugan W J,Rice J R,Sham T L.Asymptotic Analysis of Growing Plane Stain Tensile Crack in Elastic Ideally Plastic Solids. Journal of the Mechanics and Physics of Solids . 1982,p.156-166.

[4] Cao Y C,Nemat-Nasser S.Dynamic fields near crack tip growing in an elastic-perfectly-plastic solid. Mechanics of Materials.1983,p.565-574.

[5] Achenbach J D,Dunayevsky V.Fields near a rapidly propagating crack-tip in an elastic perfectlyplastic material. Journal of the Mechanics and Physics of Solids,1981,p.687-699. 
[6] Xinzheng Lu.Studies on FRP-Concrete Interface [D],BeiJing:2004,10,p. 65-66.

[7] Qingfen Li. Fracture Mechanics and its Engineering Application[M],Harbin Engineering University Publishing Company, 2007.

[8] Bingxian Yang. Paper on Beijing International Academic Conference on Composite Material Fracture Mechanics Fracture Problems [J]. Journal of composite materials, 1984 (01),p.156-199.

[9] Zegong Zhou. Introduction of Fracture Mechanics [J]. Journal of Mechanics. 1975 (3),p. 56-59.

[10] J.R.Ricc,et al. Fracture Mechanics [J]. Mechanical progress. 1987(02),p.22-45.

[11] Yuchen Gao. Ideal Elastic-plastic Plane Stress Problem [J]. Journal of solid mechanics. 1982 (3),p.56-68.

[12] Yuchen Gao. Initiation of Crack Extension of Elastic-plastic Field [J]. Journal of solid mechanics. 1980 (01),p.668-685. 